\title{
How Should We Manage Renal Transplant Patients with Failed Allografts Who Return to Dialysis?
}

\author{
Guneet S. Kochar Anthony J. Langone \\ Vanderbilt University School of Medicine, Nashville, TN, USA
}

\section{Keywords}

Kidney transplant failure · Dialysis · Immunosuppression • Nephrectomy

\begin{abstract}
Patients with failing renal allografts often progress to endstage renal disease, unlike transplant-naive chronic kidney disease patients, in an accelerated and unpredictable manner. The variability of this population renders decisions regarding placement of permanent dialysis access more difficult. Since patients with failed allografts who return to dialysis experience higher rates of morbidity and mortality, recognition and optimization of factors that will improve survival outcomes and quality of life are tantamount to longer term success. We provide guidelines on several topics such as immunosuppression withdrawal and determination of nephrectomy need versus retaining the allograft based on the current literature and our copious single-center experience.

(c) 2020 S. Karger AG, Basel
\end{abstract}

\section{Background}

There is not a common agreement or universal protocol to follow when patients have a failing renal transplant and ultimately return to dialysis. Studies regarding the many aspects of care for this population are limited. Patients with failed allografts have a higher mortality than transplant-naive patients who initiate dialysis. This review summarizes important considerations for nephrologists to ponder as they provide most of the clinical care to this burgeoning high-risk population.

\section{Kidney Transplantation Status}

Kidney transplantation is the preferred modality of renal replacement therapy for appropriate patients with end-stage renal disease. Successful transplant patients experience lower morbidity and mortality rates and improved quality of life versus those who remain on dialysis. Patients with failed renal transplant have a greater than threefold risk of death compared to matched controls with functioning allografts. Cardiovascular events, infections, malignancy, and inflammation from graft dysfunction have been implicated as the reasons for higher illness and mortality in this high-risk population $[1,2]$.

As record numbers of renal transplantation surgeries are performed year on year with $>33,000$ last year, the incidence of patients with failed allografts returning to dialysis is increasing as well and exceeds 7,000 last year. Failed allograft patients who have been relisted on the National transplant waiting list exceed 12,500 or nearly $14 \%$ of all prevalent patients on the list. With the bur-

\section{KARGER}

(C) 2020 S. Karger AG, Basel

E-Mail karger@karger.com

www.karger.com/bpu
Anthony I. Langone, MD, FAST

Vanderbilt University School of Medicine

116121 st Avenue South S-3223 Medical Center North

Nashville, TN 37232-2372 (USA)

E-Mail anthony.langone@vumc.org 
geoning of this intricate and high-risk population, the need for how to best manage these patients to achieve optimal outcomes is glaring.

\section{Failing Allograft Management}

The British Transplantation Society has set forth guidelines regarding the care of the failing transplant [3], and there have been recent review articles espousing the importance of creating an organized pathway for safe and timely return to dialysis for patients with a failing allograft [4]. We will further discuss the important factors each nephrologist should address in their patients with failing allografts who are approaching a return to dialysis.

While ruling out reversible causes of allograft failure, appropriate planning and management of the failing allograft can be neglected or delayed. Patients are hesitant to acknowledge their transplant may be failing and hesitant to return to dialysis. A directed discussion with patients regarding their likely need for dialysis is an important first step. Management of hypertension, nutrition, bone-mineral disease, anemia, and future dialysis choice, including timely access placement, is often delayed or neglected in this population and should not differ much in strategy from chronic kidney disease (CKD) patients [5]. Where the CKD population usually progresses toward end-stage renal disease at a predictable rate if several longitudinal creatinine values are known, transplant patients experience frequent kidney injuries from a myriad of etiologies including rejections, infections, and acute drug toxicities that often accelerate their allograft's demise [6]. Once a patient appears to be moving toward dialysis, careful attention to factors as detailed below that are thought to accelerate kidney dysfunction may slow the rate of renal progression. Invoking a multidisciplinary approach to care with dieticians, social workers, and vascular access surgeons, while increasing the frequency of office visits with the nephrologist, improves the likelihood of a smooth transition to dialysis. Early referral for eligible patients to renal transplant centers will likely lead to earlier placement on the active waiting list and accumulation of waiting time before they require dialysis [4].

Hypertension control is an important goal for reducing renal disease progression and lowering cardiovascular events and resultant mortality [7]. Kidney disease improving global outcomes (KDIGO) guidelines regarding blood pressure management in patients with a transplanted kidney are similar to the British Transplantation Society guidelines. The KDIGO guidelines recommend treatment if systolic blood pressure rises above 130 or di- astolic blood pressure rises above 80 [8]. Data regarding the best agent for blood pressure management in this population are lacking. There is a suggestion that dihydropyridine calcium channel blockers may obviate some of the arterial vasoconstriction of calcineurin inhibitors and lower chronic ischemic, while RAAS blockade agents will reduce proteinuria; their benefit beyond blood pressure control is not as established as in the CKD population. A good strategy is to choose agents based on cost, tolerance, and comorbidities, for example, beta blockers in known coronary artery disease patients.

Hyperlipidemia control is important to prevent major adverse cardiac events. Over one-third of deaths were due to major adverse cardiac events in the first 3 years after graft failure and initiation of dialysis. Fluvastatin, pravastatin, and rosuvastatin do not utilize the CYP3A isoenzyme for metabolism, which could lead to less interaction with concurrent use of immunosuppressants and lower rates of rhabdomyolysis.

Management of CKD-related mineral bone disorders per the updated KIDGO guidelines from 2017 recommends the use of testing of bone density if it will change management and should match the management of matched CKD patients without an allograft. Aggressive dietary changes to limit serum phosphorus including the use of phosphorous binders will also limit parathyroid hypertrophy and the resulting bone disease of hyperparathyroidism. Vitamin D analogs and calcimimetics should be used as necessary in this population as in the transplant-naive CKD population.

Albuminuria development in the transplanted organ has been associated independently with allograft failure, cardiovascular events, and death. Based on the FAVORIT trial [9], when estimated glomerular filtration rate (GFR) and albuminuria were used together, lower estimated GFR and higher albuminuria predicted a higher rate of major cardiovascular events $(2.7 \times)$ and heart failure $(3 \times)$. There has not been a demonstrated benefit of RAAS inhibition slowing renal allograft failure in proteinuric transplant patients.

A randomized controlled trial, addressing anemia in renal transplant patients, has suggested a benefit of maintaining a normal hemoglobin range (12.5-13.5 g/dL) with resultant slowing of renal progression in transplant patients with CKD [10]. Perhaps due to higher levels of inflammation, transplant patients typically require higher doses of erythropoietin-stimulating agents when compared to similar CKD patients without transplants. Maintaining adequate iron stores and earlier consideration with erythropoietin-stimulating agents is advised. After 
initiation of dialysis, patients with failed transplants had no significant increase in mortality if hemoglobin fell below $10 \mathrm{~g} / \mathrm{dL}$ [2], so anemia management should be the same as other end-stage patients.

Depending on the length of time a patient has been transplanted, nuances of dialysis may have changed since their prior experience. Treatment options will need to be discussed as if this was their first experience with endstage disease. It is important to review their past medical history as some patients will have vascular access issues or a sclerosed peritoneal cavity that if known will direct the modality discussion in the proper direction. Peritoneal dialysis was shown to have an improvement in mortality during the first year of dialysis after allograft failure; however, overall mortality was equivalent to hemodialysis over the total 3-year study period. If the peritoneum is intact despite prior surgeries, there was not an increased rate of peritonitis in failed transplant- versus incident transplant-naive dialysis patients when performing peritoneal dialysis [11]. Residual renal function loss in the allograft failure population maybe accelerated versus transplant-naive patients requiring more frequent peritoneal dialysis prescription changes and ultimately dialysis fatigue leading to an earlier transition to hemodialysis [12].

The unpredictable and erratic decline in transplant renal function makes timing of dialysis access placement more challenging than in native kidney CKD progression [13]. Prior arteriovenous access is unlikely to remain patent in transplanted patients although patients should be encouraged to protect their fistulas after transplantation as much as possible. As a result of these factors, there is an unfortunate increased reliance on central venous catheters when initiating dialysis in failed allograft patients [14]. Use of catheters, tunneled or nontunneled, led to an increase in all-cause mortality for patients who were starting dialysis after allograft failure. Early referral to the access surgeon after in-depth discussions with the patients regarding dialysis options is paramount to obtaining permanent access prior to dialysis initiation.

Starting dialysis in the setting of allograft failure is not standardized, though there are some data that may suggest higher mortality in those patients started on renal replacement therapy at a GFR $>10 \mathrm{~mL} / \mathrm{min}$ [15]. Residual renal function loss is faster in those with failed allografts, and onset of uremic symptoms maybe experienced earlier than in the transplant-naive population.

Immunosuppression medications are the cornerstone of transplantation; however, the balance between the benefits of maintaining versus discontinuing immunosuppression in the failed allograft is important to consider. The benefits of maintaining immunosuppression include decreasing sensitization, lowering the risk of acute rejection and need for nephrectomy [21], and lower risk of adrenal insufficiency possibly longer preservation of residual renal function, which is important in the consideration of peritoneal dialysis. The risks of maintaining immunosuppression are infections, malignancies, cardiovascular disease, new onset diabetes or worsening of pre-existing diabetic control, and avoidance of complications from long-term steroid use [6]. Prior to dialysis onset, patients should be maintained on their regular immunosuppression regimen and there should be consideration of maintaining this regimen if the patient has a high likelihood of being transplanted within the next year, for example, living donation. There is no consensus on how to taper off immunosuppression. We historically remove antimetabolic agents at the time of declaration of graft failure followed by tapering the calcineurin or mTor inhibitors over the following month. Antimetabolite agents are more often the first to be removed. Over half of 93 US transplant centers remove antimetabolite agents initially, while almost $40 \%$ will taper the calcineurin inhibitors first [16]. Over one-fifth will maintain patients on prednisone indefinitely, while over $70 \%$ will remove immunosuppressive agents completely by 1 year after dialysis initiation. When compared with a gradual withdrawal, rapid decrease in immunosuppression increases the risk of class I human leukocyte antigen (HLA) antibodies (panel reactive antibodies). This leads to increased donor-specific antibody production, an increase in class II HLA antibodies [17], and possibly more difficulty finding acceptable donors going forward [22].

Nephrectomy is not a low-risk procedure and is not performed routinely in patients with a failed allograft. Indications for nephrectomy include an acute immunologic rejection, which often presents as graft pain, hematuria, hypertension, and anemia [18, 19]. Nonimmunologic graft dysfunction syndrome from recurrent infections, obstruction, or stones is also an indication for surgery [20]. A nephrectomy may also be required to create space for future transplants.

A multidisciplinary approach with early action and frequent intervention leading to comprehensive care is key to mitigating the high mortality experienced by patients with failed allografts who return to dialysis. Although more work needs to be done, following guidelines set forth by the British Transplantation Society, or a checklist model as proposed by Agrawal and Pavlakis [4], is more likely than a nonstructured approach to improve the transition of these patients to dialysis. A close relationship between community nephrologists who will 
manage this population's dialysis needs and referring renal transplant center is likely to improve immunosuppression management and increase the patient's opportunity for re-transplantation.

\section{Acknowledgment}

None.

\section{Statement of Ethics}

No ethical issues, no patients were involved in this manuscript.

\section{Disclosure Statement}

G.S.K. and A.J.L. declared no conflicts of interest.

\section{Funding Sources}

No funding sources were used in the preparation of this manuscript.

\section{Author Contributions}

G.S.K. and A.J.L.: equally participated in preparation, manuscript writing, and critical review of final preparation.

\section{References}

1 Gill JS, Abichandani R, Kausz AT, Pereira BJ. Mortality after kidney transplant failure: the impact of non-immunologic factors. Kidney Int. 2002 Nov;62(5):1875-83.

2 Brar A, Markell M, Stefanov DG, Timpo E, Jindal RM, Nee R, et al. Mortality after Renal Allograft Failure and Return to Dialysis. Am J Nephrol. 2017;45(2):180-6.

3 Andrews PA; Standards Committee of the British Transplantation Society. Summary of the British Transplantation Society Guidelines for Management of the Failing Kidney Transplant. Transplantation. 2014 Dec;98(11):1130-3.

4 Agrawal N, Pavlakis M. Caring for the patient with a failing allograft: challenges and opportunities. Curr Opin Organ Transplant. 2019 Aug;24(4):416-23.

5 Huml AM, Sehgal AR. Hemodialysis Quality Metrics in the First Year Following a Failed Kidney Transplant. Am J Nephrol. 2019; 50(3):161-7.

6 Lea-Henry T, Chacko B. Management considerations in the failing renal allograft. Nephrology (Carlton). 2018 Jan;23(1):12-9.

7 Malhotra R, Katz R, Weiner DE, Levey AS, Cheung AK, Bostom AG, et al. Blood Pressure, Chronic Kidney Disease Progression, and Kidney Allograft Failure in Kidney Transplant Recipients: A Secondary Analysis of the FAVORIT Trial. Am J Hypertens. 2019 Aug;32(9):816-23.

8 Cheung AK, Chang TI, Cushman WC, Furth SL, Ix JH, Pecoits-Filho R, et al.; Conference Participants. Blood pressure in chronic kidney disease: conclusions from a Kidney Disease: Improving Global Outcomes (KDIGO) Controversies Conference. Kidney Int. 2019 May;95(5):1027-36.
9 Weiner DE, Park M, Tighiouart H, Joseph AA, Carpenter MA, Goyal N, et al. Albuminuria and Allograft Failure, Cardiovascular Disease Events, and All-Cause Death in Stable Kidney Transplant Recipients: A Cohort Analysis of the FAVORIT Trial. Am J Kidney Dis. 2019 Jan;73(1):51-61.

10 Tsujita M, Kosugi T, Goto N, Futamura K, Nishihira M, Okada M, et al. The effect of maintaining high hemoglobin levels on long-term kidney function in kidney transplant recipients: a randomized controlled trial. Nephrol Dial Transplant. DOI: 10.1093/ndt/gfy365.

11 Perl J, Dong J, Rose C, Jassal SV, Gill JS. Is dialysis modality a factor in the survival of patients initiating dialysis after kidney transplant failure? Perit Dial Int. 2013 Nov-Dec; 33(6):618-28

12 Schiffl H, Mücke C, Lang SM. Rapid decline of residual renal function in patients with late renal transplant failure who are re-treated with CAPD. Perit Dial Int. 2003 Jul-Aug; 23(4):398-400.

13 Sleiman J, Garrigue V, Vetromile F, Mourad G. Return to dialysis after renal allograft loss: is dialysis treatment initiated too late? Transplant Proc. 2007 Oct;39(8):2597-8.

14 Laham G, Pujol GS, Vilches A, Cusumano A, Diaz C. Nonprogrammed Vascular Access Is Associated With Greater Mortality in Patients Who Return to Hemodialysis With a Failing Renal Graft. Transplantation. 2017 Oct; 101(10):2606-11.

15 Molnar MZ, Streja E, Kovesdy CP, et al. Estimated glomerular filtration rate at reinitiation of dialysis and mortality in failed kidney transplant recipients. Nephrol Dial Transplant. 2012 Jul;27(7):2913-21.
16 Bayliss GP, Gohh RY, Morrissey PE, Rodrigue JR, Mandelbrot DA. Immunosuppression after renal allograft failure: a survey of US practices. Clin Transplant. 2013 Nov-Dec;27(6) 895-900.

17 Nimmo AM, McIntyre S, Turner DM, Henderson LK, Battle RK. The Impact of Withdrawal of Maintenance Immunosuppression and Graft Nephrectomy on HLA Sensitization and Calculated Chance of Future Transplant. Transplant Direct. 2018 Nov;4(12): e409.

18 Wang K, Xu X, Fan M, Qianfeng Z. Allograft nephrectomy vs. no-allograft nephrectomy for renal transplantation: a meta-analysis. Clin Transplant. 2016 Jan;30(1):33-43.

19 Chowaniec Y, Luyckx F, Karam G, Glemain P, Dantal J, Rigaud J, et al. Transplant nephrectomy after graft failure: is it so risky? Impact on morbidity, mortality and alloimmunization. Int Urol Nephrol. 2018 Oct;50(10): 1787-93.

20 Ayus JC, Achinger SG, Lee S, Sayegh MH, Go AS. Transplant nephrectomy improves survival following a failed renal allograft. J Am Soc Nephrol. 2010 Feb;21(2):374-80.

21 Del Bello A, Congy-Jolivet N, Sallusto F, Guilbeau-Frugier C, Cardeau-Desangles I, Fort M, et al. Donor-specific antibodies after ceasing immunosuppressive therapy, with or without an allograft nephrectomy. Clin J Am Soc Nephrol. 2012 Aug;7(8):1310-9.

22 Lenaers J, Christiaans M, van Heurn E, van Hooff $\mathrm{H}$, van den Berg-Loonen E. Frequent but late donor-directed antibody formation after kidney transplantectomy within one month after grafting. Transplantation. 2006 Feb;81(4):614-9. 